\title{
Vers l'intégration de la santé environnementale dans les pratiques quotidiennes de soins
}

\author{
Towards the Integration of Environmental in Daily Care Practices
}

\author{
M.-P. Sauvant-Rochat $\cdot$ C. Marie $\cdot$ F. Vendittelli \\ (C) Lavoisier SAS 2017
}

Pollution atmosphérique, pollution de l'air intérieur, contaminations des eaux par des résidus de médicaments, des métaux lourds, pollution sonore, ondes électromagnétiques, nanoparticules, etc. Ces mots font de plus en plus souvent la une des médias. De ce fait, notre société peut être confrontée à une vision anxiogène de notre cadre de vie [1]. Face à la multiplicité des messages d'alerte, tout individu est en droit de se demander si le risque pour sa santé est réel, et surtout ce qu'il doit faire pour se protéger. Ces interrogations sont particulièrement présentes chez les femmes enceintes, pour elles-mêmes et pour leur futur enfant.

Dans ce contexte, tout professionnel de santé en périnatalité peut être perçu comme un référent. Il se doit alors d'informer sur le lien entre environnement et santé, de dédramatiser quand il le faut ou au contraire de guider vers des comportements favorables à la santé dans d'autres situations [2]. Pour cela, il doit être formé, compétent et engagé dans une démarche de promotion de la santé environnementale (ou environmental health).

\footnotetext{
M.-P. Sauvant-Rochat $(\bowtie) \cdot C$. Marie $\cdot$ F. Vendittelli Université Clermont Auvergne, CNRS, Sigma, Institut Pascal, Axe TGI-PEPRADE, 28 place Henri-Dunant BP 38, F-63001 Clermont-Ferrand, France

e-mail : m-pierre.sauvant-rochat@uca.fr
}

\section{M.-P. Sauvant-Rochat}

Université Clermont Auvergne, UFR pharmacie, département santé publique et environnement, F-63001 Clermont-Ferrand, France

\section{Marie}

CHU de Clermont-Ferrand, pôle santé publique, service de biostatistique, 58 rue Montalembert, F-63003 Clermont-Ferrand cedex 1, France

F. Vendittelli

CHU de Clermont-Ferrand, pôle gynécologie-obstétrique,

58 rue Montalembert, F-63003 Clermont-Ferrand cedex 1, France

AUDIPOG (Association des Utilisateurs de Dossiers informatisés en Pédiatrie, Obstétrique et Gynécologie),

RTH Laennec Medical University, 7 rue Guillaume Paradin,

F-69372 Lyon cedex 08, France
Si la promotion de la santé environnementale est présente dans les pays anglosaxons, elle est encore balbutiante en France malgré les Plans Nationaux Santé Environnement (PNSE1 - 2004-2008, PNSE2 - 2009-2013 et PNSE3 2105-2109) [3-5] et les incitations à la formation pour les professionnels de santé [6].

Après un état des connaissances sur les risques pour la santé liés à la pollution atmosphérique, ou à la contamination du lait maternel, des exemples de démarche préventive et d'éducation des individus à la santé environnementale sont présentés dans ce numéro spécial « Environnement et santé en périnatalité », et incitent à une réflexion collective, pour que le xxie siècle soit celui de la promotion de la santé environnementale.

L'Organisation mondiale de la Santé (OMS) a résumé de façon très pragmatique la situation actuelle : « Il faut améliorer l'environnement pour améliorer notre santé » [7]. Ne l'oublions pas!

\section{Références}

1. Beck U (2001) La société du risque. Collection Alto, Editions Aubier, $521 \mathrm{p}$

2. Di Renzo GC, Conry JA, Blake J, et al (2015) International Federation of Gynecology and Obstetrics opinion on reproductive health impacts of exposure to toxic environmental chemicals. Int J Gynaecol Obstet 131:219-25

3. Plan National Santé Environnement 1 (PNSE1 - 2004-2008). Disponible sur : http://solidarites-sante.gouv.fr/IMG/pdf/synthese_pnse12.pdf (consulté le 31/07/2017)

4. Plan National Santé Environnement 2 (PNSE2 - 2009-2013). Disponible sur : http://solidarites-sante.gouv.fr/IMG/pdf/pnse2.pdf (consulté le 31/07/2017)

5. Plan National Santé Environnement 3 (PNSE3 - 2015-2019). Disponible sur : https://www.ecologique-solidaire.gouv.fr/sites/default/ files/PNSE3_v\%20finale.pdf (consulté le 31/07/2017)

6. Arrêté du 8 décembre 2015 fixant la liste des orientations nationales du développement professionnel continu des professionnels de santé pour les années 2015 à 2018. Journal Officiel de la République Française du 17 décembre 2015, texte 41. Disponible sur Légifrance : https://www.legifrance.gouv.fr/affichTexte.do?cidTexte=JORFTEXT 000031632884\&categorieLien=id (consulté le 31/07/2017)

7. Prüss-Ustün A, Wolf J, Corvalan C, et al (2016) Preventing disease through healthy environments - A global assessment of the burden of disease from environmental risks. World Health Organization Report, $\mathrm{n}^{\circ} \mathrm{WA} 305,176 \mathrm{p}$ 